BULL. AUSTRAL. MATH. SOC.

06A 75

VOL. 19 (1978), 467-474.

\title{
The density theorem for loop near-rings
}

\section{Santhakumari}

An algebraic formulation of the density theorem for loop nearrings is presented, which generalizes the same result for nearrings.

\section{Introduction}

The algebraic and topological formulations of the density theorem are quite well known in ring theory. These results have been extended by several authors for primitive near-rings; in particular Betsch [1] proved a density theorem for 0-primitive near-rings. The aim of this paper is to generalize Betsch's result $[1,2.14]$ to the case of algebraic systems known as loop near-rings.

\section{Preliminaries}

For the definitions of loops and normal subloops see [2]. We recall [3, Definition 1.1] that a loop near-ring $N=(N,+, \cdot, 0)$ is a system where

(1) $(N,+)$ is an additive loop with identity 0 , which we denote by $N^{+}$;

(2) $(N, \cdot)$ is a semigroup;

(3) $a \cdot(b+c)=a \cdot b+a \cdot c$ for all $a, b, c$ in $N$; and

(4) $0 \cdot a=0$, for all $a \in N$.

Received 7 November 1978. The author takes this opportunity to thank her director, Professor D. Ramakotaiah, for his valuable suggestions. 
For any $a$ belonging to an additive loop we shall denote the unique right and left additive inverses of $a$ by $a_{r}$ and $a_{\eta}$, respectively. Now it is easy to verify that $a 0=0,(a b)_{r}=a b_{r},(a b)_{\eta}=a b_{\eta}$, for all $a, b$ in $N$.

Throughout this paper $N$ always stands for a loop near-ring. The identity element of $(N,+)$ will be denoted by 0 .

An $N$-loop $G$ is an additive loop $(G,+, \overline{0})$ together with a mapping $(g, n) \rightarrow g n$ of $G \times N \rightarrow G$ such that for all $n, m \in N, g \in G$,

(1) $g(n+m)=g n+g m$,

(2) $g(n m)=(g n) m$.

Then we have $g 0=\overline{0},(g n)_{r}=g n_{r}, \quad(g n)_{l}=g n_{l}$, for all $g \in G$ and $n \in N$. We abbreviate $(G,+, 0)$ by $G$. A subloop $\Delta$ of an $N$-loop $G$ is called an $N$-subloop of $G$, provided that $\Delta N \subseteq \Delta$. The $N$-subloops in $N^{+}$are called $N$-loop modules of $N . N$-loop homomorphisms and $N$-loop kernels are defined in the usual way. The quotient $N$-loop of an $N$-loop $G$ by an $N$-loop kernel $K$ will be denoted by $G / K$, where $(g+K) n=g n+K$, for all $g+K \in G / K$ and $n \in N$.

A nonempty subset $K$ of an $N$-loop $G$ is an $N$-loop kernel of $G$ if and only if

(1) $(K,+)$ is a normal subloop of $G$,

(2) for all $g \in G, k \in K, n \in N,(g+k) n+g n_{p} \in K \quad[3$, Theorem 1.11].

$N$-loop kernels of $N^{+}$are called right ideals. A right ideal $L$ of $N$ is an ideal if and only if $N L \subseteq L$. Intersection of any family of $N$-loop kernels of an $N$-loop $G$ is an $N$-loop kernel of $G$ [3, Theorem 1.14]. The set of all $N$-loop kernels of an $N$-loop forms a commutative semigroup under addition. If $G$ is an $N$-loop, then for any $g \in G$, $g N=\{g n \mid n \in N\}$ is an $N_{\text {-subloop of }} G$, and $A(g)=\{n \in N \mid g n=0\}$ is a right ideal of $N ; A(G)=\bigcap_{g \in G} A(g)^{\circ}$ is an ideal of $N . N$-loops of type $V, V$-primitive loop near-rings, $V$-primitive ideals for $V=0,1,2$ are defined in the usual way as for near-rings. For further results and information see [3]. 


\section{Algebraic formulation of the density theorem}

Our proof of the density theorem (3.16) will be based on Lemmas 3.12 and 3.15. We shall obtain these lemmas as consequences of the fundamental result (Lemma 3.9), which generalizes the same result for near-rings due to Wielandt [1, Lemma 2.9]. We start with the following

THEOREM 3.1. Let $G$ be an N-loop with an $N$-generator $g$, and $L$ a right ideal of $N$; then $g L=\{g a \mid a \in L\}$ is an $N$-loop kernel.

We first divide this theorem into several lemmas and prove one after the other.

LEMMA 3.2. $g^{L}$ is a subloop of $G$.

Proof. Let $g a, g b \in g L$. Then $g a+g b=g(a+b) \in g L$ and $g 0=\overline{0} g L$. Since $g a, g b \in G$, there exist unique elements $x_{1}$ and $x_{2}$ in $G$ such that $g b=g a+x_{1}=x_{2}+g a$. Since $a, b \in L$ and since $L$ is a subloop of $N^{+}$, there exist unique elements $a_{1}, a_{2}$ in $L$ such that $b=a+a_{1}=a_{2}+a$. Hence $g b=g a+g a_{1}=g a_{2}+g a$. Therefore, by the uniqueness of $x_{1}, x_{2}$, we have $x_{1}=g a_{1}$ and $x_{2}=g a_{2}$. Hence $x_{1}, x_{2} \in g L$. Therefore $g^{L}$ is a subloop of $G$.

LEMMA 3.3. $g L$ is a normal subloop of $G$.

Proof. Let $x \in G$ and $g a \in g L$. Then $x=g n$, where $n \in N$. Consider $x+g a=g n+g a=g(n+a)$. Since $L$ is a normal subloop of $N^{+}, n+L=L+n$ for all $n \in N$. But $n+a \in n+L$. Hence $n+a=b+n$, where $b \in L$. Then

$$
x+g a=g(n+a)=g(b+n)=g b+g n=g b+x .
$$

Therefore $x+g a \in g L+x$. Hence $x+g L \subseteq g L+x$. Similarly it can be proved that $g L+x \subseteq x+g L$. Therefore $x+g L=g L+x$ for all $x \in G$. Let $x, y \in G$ and $g a \in g L$. Then $x=g n_{1}, y=g n_{2}$, where $n_{1}, n_{2} \in N$. Now $(x+y)+g a=\left(g n_{1}+g n_{2}\right)+g a=g\left(\left(n_{1}+n_{2}\right)+a\right)$. Since $L$ is a normal subloop of $N^{+},\left(n_{1}+n_{2}\right)+L=n_{1}+\left(n_{2}+L\right)$. Now $\left(n_{1}+n_{2}\right)+a \in\left(n_{1}+n_{2}\right)+L$. Hence $\left(n_{1}+n_{2}\right)+a=n_{1}+\left(n_{2}+b\right)$, where $b \in L$. Therefore 


$$
(x+y)+g a=g\left(\left(n_{1}+n_{2}\right)+a\right)=g\left(n_{1}+\left(n_{2}+b\right)\right)=g n_{1}+\left(g n_{2}+b\right)=x+(y+g b) .
$$

Hence $(x+y)+g a \in x+(y+g L)$. Therefore $(x+y)+g L \subseteq x+(y+g L)$. The other inclusion is also verified. Therefore $(x+y)+g L=x+(y+g L)$ for all $x, y \in G$. By a similar argument it can be shown that for all $x, y \in G, g L+(y+x)=(g L+y)+x$. Therefore $g L$ is a normal subloop of $G$.

LEMMA 3.4. $g L$ is an $N$-Zoop kemez of $G$.

Proof. Let $g a \in g L, x \in G$, and $n \in N$. Let $x=g m$ where $m \in N$. Now $(x+g a) n+x n_{r}=(g m+g a) n+(g m) n_{r}=g\left((m+a) n+m n_{p}\right) \in g L$, since $L$ is a right ideal of $N$ and $a \in L$.

By Lemmas 3.1 and 3.2, $g L$ is a normal subloop of $G$. Therefore $g L$ is an $N$-loop kernel of $G$.

COROLLARY 3.5. If $G$ is an N-loop of type $I$ and $L$ a right ideal of $N$, then for each $g \in G$, either $g L=\{\overline{0}\}$ or $g L=G$.

Proof. If $g L \neq\{\overline{0}\}$, then $g N \neq\{\overline{0}\}$ and hence $g N=G$. Therefore $g$ is an $N$-generator of $G$. Since $G$ is irreducible and since $\{\overline{0}\} \neq g L$ is an $N$-loop kernel of $G$, we have $g L=G$.

COROLLARY 3.6. If $G$ is an N-Zoop of type I with $A(G)=\{0\}$ and $L \neq\{0\}$ a right ideal of $N$, then there exists an element $g \in G$ such that $g L=G$.

Proof. Since $A(G)=\{0\}$, there exists an element $g \in G$ such that $g L \neq\{\overline{0}\}$. Hence $g L=G$ (Corollary 3.5).

LEMMA 3.7. Let $G$ be an N-Loop with $A(G)=\{0\}$. If $N^{+}$is not associative then $G$ is not associative.

Proof. Since $N^{+}$is not associative, there exist $x, y, z \in N$ such that $(x+y)+z \neq x+(y+z)$. Since $A(G)=\{0\}$, there exists a $g \in G$ such that $g((x+y)+z) \neq g(x+(y+z)) ;$ for otherwise

$$
g((x+y)+z)=g(x+(y+z))
$$

for all $g \in G$ implies that $g((x+y)+z)+g(x+(y+z))_{r}=\overline{0}$ for all $g \in G$, and hence $((x+y)+z)+(x+(y+z))_{r} \in A(G)=\{0\}$. Therefore $((x+y)+z)+(x+(y+z))_{r}=0=(x+(y+z))+(x+(y+z))_{r}$; hence by 
cancellation in $N^{+},(x+y)+z=x+(y+z)$, a contradiction. Therefore: for some $g \in G, g(x+(y+z)) \neq g((x+y)+z)$; that is, $g x+(g y+g z) \neq(g x+g y)+g z$. Therefore $G$ is not associative.

LEMMA 3.8 [4, Proposition 2.1]. Let $A, B, K$ be normal subloops of an additive loop $G$; then the additive loop

$$
\bar{G}:=(A+K) \cap(B+K) /(A \cap B)+K
$$

is an abelian group.

LEMMA 3.9. Let $N$ be a loop near-ming and let $A, B, K$ be $N$-loop kermels of some $N$-loop $G$. Then the N-Zoop

$$
\bar{G}:=(A+B) \cap(B+K) /(A \cap B)+K
$$

is an abelian group, and for all $\bar{g} \in \bar{G}, n \in N$ the mapping $\bar{G}+\bar{G}: \bar{g} \rightarrow \bar{g} n$ is an endomorphism of $(\bar{G},+)$.

Proof. Let $E=(A \cap B)+K$ and $H=(A+K) \cap(B+K)$; then $\bar{G}:=H / E$ is an abelian group (Lemma 3.8). Now it is enough to show that for all $x, y \in H$ and $n \in N,(x+y) n+E=(x n+y n)+E$. Let $x, y \in H$; then $x \in A+K$ and $y \in B+K$; therefore $x=a+p$, $y=b+q$ for some $a \in A, b \in B$, and $p, q \in K$. Since $E$ is a normal subloop, $x+E=(a+p)+E=a+(p+E)=a+E$, since $p \in K$ implies $p \in E$; hence $p+E=E$. Similarly we get $y+E=b+E$. Since $a \in A$ and $A$ is an $N$-loop kernel of $G$, we have $(a+b) n+b n_{p} \in A$, and also an $\in A$. Therefore

$$
\left((a+b) n+b n_{p}\right)+A=A=\overline{0}+A=\left(b n+b n_{p}\right)+A
$$

since $G / A$ is a loop and the cancellation laws hold good in a loop, we have $(a+b) n+A=b n+A=A+b n=(A+a n)+b n=A+(a n+b n)$. Since $(a+b) n \in(a+b) n+A$, we have $(a+b) n \in A+(a n+b n)$. Since $A$ is a normal subloop of $G$, we have $(a+b) n+(a n+b n)_{r} \in\{A+(a n+b n)\}+(a n+b n)_{r}$

$$
=A+\left\{(a n+b n)+(a n+b n)_{p}\right\}=A+\overline{0}=A .
$$

By a similar argument we get $(a+b) n+(a n+b n)_{p} \in B$. Therefore $(a+b) n+(a n+b n)_{p} \in A \cap B \subseteq E$. Hence

$$
\left\{(a+b) n+(a n+b n)_{p}\right\}+E=E=\overline{0}+E=\left\{(a n+b n)+(a n+b n)_{p}\right\}+E .
$$


Since $G / E$ is a loop, we get $(a+b) n+E=(a n+b n)+E$. Therefore $(x+y) n+E=(x n+y n)+E$, since $x+E=a+E$ and $y+E=b+E$. Hence the result.

COROLLARY 3.10. Under the assumptions and with the notation of Lemma 3.9, we get $\bar{N}=N / A(\bar{G})$ is a ring.

Proof. $\bar{G}$ is an abelian group (Lemma 3.9). Further, $\bar{G}$ is a faithful $\bar{N}$-loop. Since addition in $\bar{G}$ is associative, addition in $\bar{N}$ is also associative (Lemma 3.7); consequently $(\bar{N},+)$ is a group, and hence $\bar{N}$ is a near-ring. Hence $\bar{G}$ is a faithful $\bar{N}_{\text {-group and }} \bar{G}$ is abelian. Further, for all $\bar{n} \in \bar{N}, \bar{g} \rightarrow \overline{g n}$ is an endomorphism of $(\bar{G},+$ ) (Lemma 3.9). Therefore $\bar{N}$ is a ring [1, Lemma 1.5].

COROLLARY 3.11. Let $N$ be a loop near-ring with identity and let no nonzero epimorphic image of $N$ be a ring. Then the lattice of right ideals of $N$ is distributive.

The proof is similar as in the case of near-rings [1, Corollary 2.1]. As a further consequence of Lemma 3.9 we obtain:

LEMMA 3.12. Let $N$ be a loop near-ring and $G$ an $N$-loop which is monogenic with generator $g\left(G=g^{N}\right)$ and faithful. Let $B$ and $C$ be right ideals of $N$ with the property that $B+A(g)=C+A(g)=N$ and $B \cap C \subseteq A(g)$. Then $N$ is a ring.

Proof. We form the $N$-loop

$$
\bar{G}:=[B+A(g)] \cap[C+A(g)] /(B \cap C)+A(g)=N / A(g),
$$

which is $N$-isomorphic to $G$. By Lemma $3.9, \bar{G}$ is an abelian group, and hence $G$ is an abelian group; therefore $N$ is a near-ring and $N$ induces only endomorphisms of $(G,+)$ on $G$. Since $G$ is faithful, $N$ must be a ring [1, Lemma 1.5].

Let $N$ be a 0-primitive loop near-ring. $G$ will denote a faithful $N$-loop of type 0 . We denote the set $\left\{g \in G \mid g^{N}=G\right\}$ by $C$. That is, $C$ is the set of all $N$-generators of $G$, and $C \neq \varnothing$.

LEMMA 3.13. Let $G$ be an $N$-loop of type 0 and $g \in C$. Then $A(g)$ is a maximal might ideal of $N$, and the quotient loop $N / A(g)$ is N-Zoop isomorphic to $G$.

The proof is easy and will be omitted. 
COROLLARY 3.14. If $g, g_{1} \in C$ and if $A(g) \supseteq A\left(g_{1}\right)$, then $A(G)=A\left(g_{1}\right)$.

The proof is easy and will be omitted.

LEMMA 3.15. Let $N$ be a loop near-ring which is not a ring and is o-primitive on $G$. Let $g_{1}, g_{2}, \ldots, g_{s} \in C$. Assume $A\left(g_{i}\right) \neq A\left(g_{j}\right)$ for $i \neq j$. Then for $t=1,2, \ldots, s-1$ the following statement is true:

$$
S(t)=\bigcap_{i=1}^{t} A\left(g_{i}\right) \pm A\left(g_{k}\right) \text { for all } t<k \leq s .
$$

The proof of this lemma is similar as in the case of near-rings [1, Lemma 2.13].

THEOREM 3.16. Let $N$ be a 0-primitive loop near-ring which is not a ring, and $G$ an $N$-Zoop of type 0 with $A(G)=\{0\}$. Let $g_{1}, g_{2}, \ldots, g_{k} \in C$ such that $A\left(g_{i}\right) \neq A\left(g_{j}\right)$ for $i \neq j$. For arbitrary elements $g_{1}^{\prime}, g_{2}^{\prime}, \ldots, g_{k}^{\prime}$ of $G$ there exists on $n \in N$ such that $g_{i}^{\prime}=g_{i} n$ for $i=1,2, \ldots, k$.

Proof. Since $g_{1} \in C$, there exists an element $n_{t} \in N$ such that $g_{1} n_{1}=g_{1}^{\prime}$. So the result is true for $k=1$. Assume the result for $i=1,2, \ldots, t$, where $1 \leq t \leq k-1$. Then there exists an $n_{t} \in N$ such that $g_{i} n_{t}=g_{i}^{\prime}$ for $i=1,2, \ldots, t$. We have

$$
K:=\prod_{i=1}^{t} A\left(g_{i}\right) \pm A\left(g_{i+1}\right)
$$

(Lemma 3.15). Hence $g_{t+1} K \neq\{0\}$, and since $g_{t+1} K$ is an $N$-loop kernel. of $G, g_{t+1} K=G$. Therefore there exists an element $k \in K$, such that $g_{t+1}^{\prime}=g_{t+1} k$. Also there exists a $k^{\prime} \in K$, such that $g_{t+1} n_{t}=g_{t+1} k^{\prime}$. Let $n_{t+1}=\left(n_{t}+k_{r}^{\prime}\right)+k$. Then

$$
g_{i} n_{t+1}=\left(g_{i} n_{t}+g_{i} k_{p}^{\prime}\right)+g_{i} k=g_{i} n_{t}=g_{i}^{\prime}
$$

for $i=1,2, \ldots, t$; 
$g_{t+1}^{n} t+1=\left(g_{t+1} n_{t}+g_{t+1} k_{p}^{\prime}\right)+g_{t+1} k$

$$
=\left(g_{t+1} k^{\prime}+g_{t+1} k_{r}^{\prime}\right)+g_{t+1} k=g_{t+1} k=g_{t+1}^{\prime} .
$$

Hence $g_{i} n_{t+1}=g_{i}^{\prime}$ for $i=1,2, \ldots, t+1$.

By induction the result follows.

\section{References}

[1] Gerhard Betsch, "Primitive near-rings", Math. 2. 130 (1973), 351-361.

[2] Richard Hubert Bruck, A survey of binary systems, 2nd edition

(Ergebnisse der Mathematik und ihrer Grenzgebiete, 20. SpringerVerlag, Berlin, Heidelberg, New York, 1966).

[3] D. Ramakotaiah and C. Santhakumari, "On loop near-rings", BulZ. Austral. Math. Soc. 19 (1978), 417-435.

[4] C. Santhakumari, "On quotient loops of normal subloops", Canad. Math. Bulz. (to appear).

Department of Mathematics,

Nagarjuna University,

Nagarjunanagar,

Andhra Pradesh,

India. 